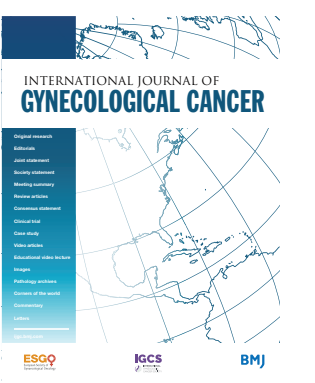

For numbered affiliations see end of article.

Correspondence to

Tamara Jones, School of Allied Health Sciences, Griffith University, Brisbane, QLD 4111, Australia; tamara.jones3@ griffithuni.edu.au

Received 29 September 2020 Revised 21 December 2020 Accepted 23 December 2020 Published Online First 18 January 2021
Check for updates

(C) IGCS and ESGO 2021. № commercial re-use. See rights and permissions. Published by BMJ.

To cite: Jones T, Sandler C, Vagenas D, et al. Int J Gynecol Cancer 2021;31:553-561.

\title{
Physical activity levels among ovarian cancer survivors: a prospective longitudinal cohort study
}

Tamara Jones (D) , ${ }^{1}$ Carolina Sandler, ${ }^{2,3}$ Dimitrios Vagenas, ${ }^{2}$ Monika Janda, ${ }^{2,4}$ Andreas Obermair, ${ }^{5}$ Sandra Hayes ${ }^{6}$

\section{HIGHLIGHTS}

- Within 2-years post-diagnosis of ovarian cancer, average physical activity levels are below recommended levels.

- More than $50 \%$ of women with ovarian cancer report a decrease or no change in physical activity levels post-diagnosis.

- Future research now needs to evaluate the safety, feasibility, and effect of exercise post-ovarian cancer.

\section{ABSTRACT}

Objective Physical activity following cancer diagnosis is associated with improved outcomes, including potential survival benefits, yet physical activity levels among common cancer types tend to decrease following diagnosis and remain low. Physical activity levels following diagnosis of less common cancers, such as ovarian cancer, are less known. The objectives of this study were to describe physical activity levels and to explore characteristics associated with physical activity levels in women with ovarian cancer from pre-diagnosis to 2 years post-diagnosis.

Methods As part of a prospective longitudinal study, physical activity levels of women with ovarian cancer were assessed at multiple time points between prediagnosis and 2 years post-diagnosis. Physical activity levels and change in physical activity were described using metabolic equivalent task hours and minutes per week, and categorically (sedentary, insufficiently, or sufficiently active). Generalized Estimating Equations were used to explore whether participant characteristics were related to physical activity levels.

Results A total of 110 women with ovarian cancer with a median age of 62 years (range 33-88) at diagnosis were included. $53-57 \%$ of the women were sufficiently active post-diagnosis, although average physical activity levels for the cohort were below recommended levels throughout the 2-year follow-up period (120-142.5min/ week). A decrease or no change in post-diagnosis physical activity was reported by $44-60 \%$ of women compared with pre-diagnosis physical activity levels. Women diagnosed with stage IV disease, those earning a lower income, those receiving chemotherapy, and those currently smoking or working were more likely to report lower physical activity levels and had increased odds of being insufficiently active or sedentary.

Conclusions Interventions providing patients with appropriate physical activity advice and support for behavior change could potentially improve physical activity levels and health outcomes.

\section{INTRODUCTION}

In recent years the efficacy of physical activity and exercise across the cancer continuum has been extensively investigated. ${ }^{12}$ While physical activity encompasses all bodily movement (including exercise), exercise is planned and structured with the intent of improving specific outcomes. ${ }^{3}$ Benefits associated with physical activity and exercise during treatment and following diagnosis have resulted in statements and guidelines from national and international organizations. ${ }^{1} 2$ Internationally endorsed physical activity guidelines recommend that cancer survivors should participate in $150 \mathrm{~min} /$ week physical activity and strength training, ${ }^{4-6}$ with calls for physical activity and exercise to be incorporated into standard cancer care. ${ }^{7}$

Physical activity has been associated with survival outcomes, including all-cause and cancer-specific mortality, with findings from a recent systematic review and meta-analysis showing improved survival outcomes in 10 cancer types for those in the highest category of post-diagnosis physical activity compared with those in the lowest physical activity category. ${ }^{8}$ In particular, preliminary evidence supports the relationship between post-diagnosis physical activity and overall survival following a diagnosis of ovarian cancer. $^{9}$ Additionally, declines in physical activity following a cancer diagnosis have been associated with poorer overall survival. ${ }^{10}$ Furthermore, the American College of Sports Medicine recently released guidelines stating that there was strong evidence in support of the benefits of exercise in the management of cancer-related health outcomes including anxiety, depression, fatigue, health-related quality of life, lymphedema, and physical function. ${ }^{1}$

Despite the evidence that participating in regular physical activity is beneficial for people with cancer, epidemiological findings suggest that physical activity levels tend to decrease following diagnosis 


\section{Original research}

and remain low, with the majority of cancer survivors not meeting recommended physical activity levels 12 months or more following diagnosis. ${ }^{11-13}$ However, the understanding of post-diagnosis physical activity patterns is largely derived from mixed cancer patient samples or patients with common cancer types such as breast and colorectal cancer. ${ }^{112}$ In contrast, physical activity levels and changes in physical activity following diagnosis for those with less common cancers, in particular cancers that confer a poorer prognosis, are less well studied. A recent review of studies evaluating physical activity levels in ovarian cancer found that most women decrease their physical activity post-diagnosis and remain insufficiently active beyond the treatment period, with the majority failing to meet physical activity recommendations. ${ }^{1314}$ However, it was also noted that these findings were largely drawn from crosssectional or retrospective studies, with few assessing physical activity prospectively and a lack of data on physical activity during the first year following diagnosis. ${ }^{13} 14$

As part of a prospective longitudinal cohort study, self-reported physical activity levels from pre-diagnosis through to 2 years postdiagnosis were collected from women with ovarian cancer. ${ }^{15}$ The objectives of this study were (1) to describe physical activity levels within the first 2 years following ovarian cancer diagnosis; (2) to describe changes in physical activity levels between pre-diagnosis and 2 years post-ovarian cancer diagnosis; and (3) to explore the characteristics associated with physical activity levels from prediagnosis to 2 years post-diagnosis in women with ovarian cancer.

\section{METHODS}

\section{Study design and subjects}

Physical activity levels from pre-diagnosis to 2 years post-diagnosis were assessed as part of a prospective longitudinal cohort study in women with ovarian cancer. ${ }^{1516}$ Women were eligible to participate if they were aged $\geq 18$ years, newly diagnosed with ovarian cancer, and treated at participating hospitals in Queensland, New South Wales and Victoria, Australia. ${ }^{1516}$

\section{Timing of data collection}

Following informed consent, baseline data were collected approximately 1 week prior to diagnostic surgery and definitive gynecological cancer diagnosis. Subsequent assessments were coordinated with the participant's scheduled hospital follow-up visits between 6 weeks and 2 years post-diagnosis. Follow-up schedules differed between participants, therefore depending on the timing of the participant's follow-up appointments, assessments were conducted a maximum of 10 times over the study period (ie, at 6 weeks and 3, 6, 9, 12, 15, 18, 21, and 24 months post-diagnosis).

\section{Data collection}

Physical activity levels were assessed using the self-report Active Australia Survey, for which good to excellent reliability and acceptable validity have been reported. ${ }^{17}$ Women were asked to report the frequency and duration of activity completed in the previous week (number of sessions and time spent in each activity), including walking, moderate- and vigorous-intensity physical activity, from which min/week of each physical activity type was calculated. 'Total Activity' per week was calculated based on the sum of moderate(including walking) and vigorous-intensity activity (weighted by two). ${ }^{17}$ Total metabolic equivalent task (MET) hours/week were then calculated using $\mathrm{min} /$ week of moderate- and vigorousintensity physical activity, with MET values of 4.0 and 8.0 assigned to moderate- and vigorous-intensity activity, respectively. ${ }^{18}$ Weekly MET hours were calculated by multiplying the duration of activity per week by the assigned MET value, with Total MET hours/week calculated based on the sum of moderate- and vigorous-intensity activity. Using Total MET hours/week, women were classified into physical activity categories: 'Sedentary' (0 MET hours/week); 'Insufficiently Active' (<7.5 MET hours/week), or 'Sufficiently Active' ( $\geq 7.5$ MET hours/week), according to the minimum energy expenditure of global physical activity recommendations ( $150 \mathrm{~min} /$ week of moderate-intensity physical activity). ${ }^{419}$

Participant characteristics including demographic information, socioeconomic and marital status, and lifestyle behaviors were collected via a participant-administered questionnaire at baseline. Clinical information concerning diagnosis and treatment were abstracted by trained research nurses from medical records at baseline and at the 2-year follow-up.

\section{Statistical methods}

Due to differences in the number and timing of follow-up visits for each participant, follow-up visits were grouped into time phases for analysis of physical activity data: baseline (T1; pre-definitive diagnosis), 6 weeks to 3 months (T2), 6 months to 1 year (T3), and 15 months to 2 years post-diagnosis (T4). For T2-T4, physical activity measures were averaged if multiple surveys were completed within each time phase.

Physical activity levels were described at each time phase using min/week and MET hours/week, with both mean and medians presented due to the skewed nature of the data. Change in physical activity levels were described using continuous change (Total Activity and Total MET hours/week at T2-T4 minus baseline levels), as well as categorial change. Based on values previously reported in the literature, a clinically significant change in physical activity was determined a priori as a difference of $30 \mathrm{~min}$ in Total Activity per week or 3 METs in Total MET hours/week. ${ }^{2021}$ Participants were then categorized as having 'Increased', 'Decreased' or reported 'No Change' in physical activity from baseline to T2-T4.

Using all available data from $\mathrm{T} 1-\mathrm{T} 4$, linear and binary logistic Generalized Estimating Equations were used to explore the relationship between physical activity levels (continuous; Total MET hours/week and categorical; 'Sufficiently Active' vs 'Insufficiently Active', respectively) and individual baseline personal, diagnostic, treatment, and behavioral characteristics. Characteristics of theoretical importance (based on previous literature), as well as statistical $(p<0.05)$ or clinical significance (3 MET hours/ week difference between groups or $\mathrm{OR}$ of $\leq 0.6$ or $\geq 1.5$ ) were then included in separate models, adjusting for age and body mass index at diagnosis. ${ }^{1520}{ }^{21}$ When physical activity levels were assessed as a continuous variable, the effect size of the relationship was described using beta values and standard errors for continuous independent variables and estimated marginal means and standard errors for categorical independent variables. When physical activity was assessed as a categorical variable, relationships with independent variables were described using ORs and $95 \%$ confidence intervals. 


\begin{tabular}{|c|c|c|c|}
\hline \multicolumn{4}{|l|}{ Characteristics $(n=110)$} \\
\hline Age, years & & Menstrual status, $\mathrm{n}(\%)$ & \\
\hline Mean \pm SD & $60.72 \pm 10.68$ & Pre-/peri-menopausal & $18(16.4)$ \\
\hline Range & $33-88$ & Post-menopausal & $92(83.6)$ \\
\hline Weight, kg & & Surgery, n (\%) & \\
\hline Median (range) & $68.75(45.0-136.8)$ & Laparotomy & $96(87.3)$ \\
\hline Body mass index (BMI), $\mathrm{kg} / \mathrm{m}^{2}$ & & Lymph node dissection, $\mathrm{n}(\%)$ & \\
\hline Median (range) & $25.8(17.5-52.1)$ & Yes & $42(38.2)$ \\
\hline BMI categories, n (\%) & & Chemotherapy, n (\%) & \\
\hline Underweight/normal & $43(39.1)$ & Adjuvant \pm neoadjuvant & $88(80.0)$ \\
\hline Overweight & $36(32.7)$ & No chemotherapy & 17 (15.5.) \\
\hline Obese & $29(26.4)$ & Unknown & $5(4.5)$ \\
\hline Unknown & $2(1.8)$ & & \\
\hline Stage, n (\%) & & Highest education, n (\%) & \\
\hline Stage I & $26(23.6)$ & Grade 12 or below & $66(60.0)$ \\
\hline Stage II & $14(12.7)$ & Trade/University & $28(25.5)$ \\
\hline Stage III & $53(48.2)$ & Other & $10(9.1)$ \\
\hline Stage IV & $14(12.7)$ & Unknown & $6(5.5)$ \\
\hline Unknown & $3(2.7)$ & & \\
\hline Histological type, n (\%) & & Employment status, n (\%) & \\
\hline Epithelial & $101(91.8)$ & Full-time/part-time/casual & $25(22.7)$ \\
\hline Other & $5(4.5)$ & Retired/home duties & $66(60.0)$ \\
\hline \multirow[t]{2}{*}{ Unknown } & $4(3.6)$ & Other & $13(11.8)$ \\
\hline & & Unknown & $6(5.5)$ \\
\hline ECOG status, n (\%) & & Marital status, n (\%) & \\
\hline 0 & $92(83.6)$ & Married/de facto & $66(60.0)$ \\
\hline 1 & $16(14.5)$ & Not married & $37(33.6)$ \\
\hline \multirow[t]{2}{*}{2} & $2(1.8)$ & Other & $1(0.9)$ \\
\hline & & Unknown & $6(5.5)$ \\
\hline Smoking, n (\%) & & Household income, n (\%) & \\
\hline Never/past & $95(86.4)$ & $\leq \$ 60000$ & $71(64.5)$ \\
\hline Current & $11(10.0)$ & $>\$ 60000$ & $19(17.3)$ \\
\hline Unknown & $4(3.6)$ & Unknown & 20 (18.2) \\
\hline \multicolumn{4}{|l|}{ Birth country, n (\%) } \\
\hline Australia & $79(71.8)$ & & \\
\hline Other & $30(27.3)$ & & \\
\hline Unknown & $1(0.9)$ & & \\
\hline
\end{tabular}

\section{RESULTS}

As part of the wider study, a total of 110 women with ovarian cancer completed baseline assessment, at least one follow-up assessment, and provided data on at least one outcome such as physical activity or lymphedema. ${ }^{15} 16$ The number of participants who contributed physical activity data and were included in this analysis ranged from 84 to 98 (T1: $n=94 ; T 2: n=98 ; T 3: n=94 ; T 4$ : $\mathrm{n}=84$ ). Participant demographics are shown in Table 1. Overall, the sample had an average age of $61 \pm 11$ years and a median body mass index of $26 \mathrm{~kg} / \mathrm{m}^{2}$ (range $18-52 \mathrm{~kg} / \mathrm{m}^{2}$ ). The majority were diagnosed with late stage disease $(n=67,61 \%)$, epithelial cancer $(\mathrm{n}=101,92 \%)$, and had received chemotherapy $(\mathrm{n}=88,80 \%)$, with a laparotomy being the most common surgical approach $(\mathrm{n}=96$, $87 \%)$.

\section{Physical activity levels}

Weekly physical activity levels from T1 to T4 are shown in Table 2. Weekly minutes for walking and moderate-intensity activities were lowest at baseline before increasing from $\mathrm{T} 1$ to $\mathrm{T} 3$ and then plateauing between $\mathrm{T} 3$ and T4. This trend was reflected in 'Total Activity' (min/week) and 'Total MET hours/week'. 'Vigorous gardening or housework' showed an upward trend across the 2-year follow-up period and mean 'Vigorous physical activity' returned to baseline levels by $\mathrm{T} 3$ and $\mathrm{T} 4$, following a decrease at 


\section{Original research}

Table 2 Weekly physical activity reported by women with ovarian cancer at each time point

\begin{tabular}{|c|c|c|c|c|}
\hline & T1 $(n=94)$ & T2 $(n=98)$ & T3 $(n=94)$ & T4 $(n=84)$ \\
\hline \multicolumn{5}{|c|}{ Walking for recreation (min/week) } \\
\hline Median (min, max) & $30.00(0.00,480.00)$ & $52.50(0.00,820.00)$ & $60.00(0.00,595.00)$ & $53.75(0.00,840.00)$ \\
\hline Mean (SE) & $75.92(11.56)$ & $86.32(11.94)$ & $97.65(11.53)$ & 96.67 (15.39) \\
\hline \multicolumn{5}{|c|}{ Walking for exercise (min/week) } \\
\hline Median (min, max) & $30.00(0.00,420.00)$ & $45.00(0.00,840.00)$ & $56.67(0.00,400.00)$ & $35.00(0.00,345.00)$ \\
\hline Mean (SE) & $59.74(9.33)$ & 77.67 (11.59) & $88.88(9.78)$ & $67.76(9.23)$ \\
\hline \multicolumn{5}{|l|}{ All walking (min/week) } \\
\hline Median (min, max) & $77.50(0.00,770.00)$ & $120.00(0.00,1090.00)$ & $126.25(0.00,653.33)$ & $101.67(0.00,870.00)$ \\
\hline Mean (SE) & $128.60(17.06)$ & $163.08(19.42)$ & $181.32(18.19)$ & $164.44(21.08)$ \\
\hline \multicolumn{5}{|c|}{$\begin{array}{l}\text { Other moderate physical activity (min/ } \\
\text { week) }\end{array}$} \\
\hline Median (min, max) & $0.00(0.00,400.00)$ & $0.00(0.00,322.50)$ & $0.00(0.00,300.00)$ & $0.00(0.00,380.00)$ \\
\hline Mean (SE) & $13.44(7.07)$ & $13.06(4.72)$ & $31.36(7.08)$ & $35.47(7.90)$ \\
\hline \multicolumn{5}{|c|}{ All moderate activity (min/week) } \\
\hline Median (min, max) & $85.00(0.00,830.00)$ & $120.00(0.00,1412.50)$ & $135.83(0.00,855.00)$ & $127.50(0.00,940.00)$ \\
\hline Mean (SE) & $140.10(19.02)$ & $174.04(21.49)$ & $211.32(21.55)$ & $197.47(24.26)$ \\
\hline \multicolumn{5}{|c|}{$\begin{array}{l}\text { Vigorous gardening or housework (min/ } \\
\text { week) }\end{array}$} \\
\hline Median (min, max) & $0.00(0.00,300.00)$ & $0.00(0.00,840.00)$ & $14.33(0.00,420.00)$ & $20.00(0.00,820.00)$ \\
\hline Mean (SE) & $21.90(5.68)$ & $51.20(12.57)$ & $58.13(9.39)$ & $64.87(14.77)$ \\
\hline \multicolumn{5}{|c|}{ Vigorous physical activity (min/week) } \\
\hline Median (min, max) & $0.00(0.00,540.00)$ & $0.00(0.00,200.00)$ & $0.00(0.00,435.00)$ & $0.00(0.00,315.00)$ \\
\hline Mean (SE) & $20.12(9.17)$ & $8.91(2.98)$ & $21.02(6.12)$ & $21.85(6.10)$ \\
\hline \multicolumn{5}{|c|}{ Total activity (min/week) } \\
\hline Median (min, max) & $90.00(0.00,1345.00)$ & $120.00(0.00,1517.00)$ & $140.00(0.00,1033.33)$ & $142.50(0.00,1470.00)$ \\
\hline Mean (SE) & $175.26(29.18)$ & $189.88(24.62)$ & $251.53(26.52)$ & $238.26(32.73)$ \\
\hline \multicolumn{5}{|l|}{ Total MET hours/week } \\
\hline Median (min, max) & $6.00(0.00,89.67)$ & $8.00(0.00,101.17)$ & $9.33(0.00,68.89)$ & $9.50(0.00,98.00)$ \\
\hline Mean (SE) & $11.68(1.95)$ & $12.66(1.64)$ & $16.77(1.77)$ & $15.88(2.18)$ \\
\hline
\end{tabular}

T1: baseline; T2: 6 weeks to 3 months post-diagnosis; T3: 6 months to 1 year post-diagnosis; T4: 15 months to 2 years post-diagnosis.

MET, metabolic equivalent task.

T2. At 2 years post-diagnosis (T4), average physical activity levels were $142.50 \mathrm{~min} /$ week ('Total Activity') and 9.50 MET hours/week.

Table 3 shows the proportion of women meeting physical activity guidelines based on Total MET hours/week. Between T1 and T4, 9-19\% of women were 'Sedentary' and $29-37 \%$ were 'Insufficiently Active'. Pre-diagnosis (T1; baseline), $40 \%$ of women were categorized as being 'Sufficiently Active', whereas by T4, 56\% reported physical activity levels consistent with meeting physical activity guidelines. Data presented in Table 4 suggest that the average change between T1 and T2-T4 was positive-that is, on average women increased physical activity levels between time of diagnosis and 2 years post-diagnosis. When women were categorized according to change in physical activity levels based on Total MET hours/week, $60 \%, 44 \%$, and $53 \%$ reported either 'No Change' or 'Decreased' physical activity levels between $\mathrm{T} 1$ and T2-T4, respectively. Figure 1 shows the proportion of women who

Table 3 Proportion of women meeting physical activity guidelines and corresponding total MET hours/week at each time point

\begin{tabular}{|c|c|c|c|c|c|c|c|c|}
\hline & \multicolumn{2}{|l|}{$T 1(n=91)$} & \multicolumn{2}{|l|}{ T2 $(n=97)$} & \multicolumn{2}{|l|}{ T3 $(n=92)$} & \multicolumn{2}{|l|}{ T4 $(n=83)$} \\
\hline & N (\%) & $\begin{array}{l}\text { Median (min, } \\
\text { max) }\end{array}$ & N (\%) & $\begin{array}{l}\text { Median (min, } \\
\text { max) }\end{array}$ & N (\%) & $\begin{array}{l}\text { Median (min, } \\
\text { max) }\end{array}$ & N (\%) & $\begin{array}{l}\text { Median (min, } \\
\text { max) }\end{array}$ \\
\hline $\begin{array}{l}\text { Sedentary } \\
\text { (0 MET hours/week) }\end{array}$ & $18(19.1)$ & $\begin{array}{l}0.00(0.00 \\
0.00)\end{array}$ & $10(10.2)$ & $0.00(0.00,0.00)$ & $8(8.5)$ & $\begin{array}{l}0.00(0.00 \\
0.00)\end{array}$ & $12(14.3)$ & $\begin{array}{l}0.00(0.00 \\
0.00)\end{array}$ \\
\hline $\begin{array}{l}\text { Insufficiently Active } \\
\text { (<7.5 MET hours/week) }\end{array}$ & $35(37.2)$ & $\begin{array}{l}3.33(0.27, \\
7.33)\end{array}$ & $35(35.7)$ & $4.00(0.33,7.33)$ & 30 (31.9) & $\begin{array}{l}4.33(0.67, \\
7.33)\end{array}$ & $24(28.6)$ & $\begin{array}{l}3.42(0.50 \\
6.78)\end{array}$ \\
\hline $\begin{array}{l}\text { Sufficiently Active } \\
\text { ( } \geq 7.5 \text { MET hours/week) }\end{array}$ & $38(40.4)$ & $\begin{array}{l}14.00(8.00 \\
89.67)\end{array}$ & $52(53.1)$ & $\begin{array}{l}15.00(7.57 \\
101.17)\end{array}$ & $54(57.4)$ & $\begin{array}{l}24.00(7.67 \\
68.89)\end{array}$ & $47(56.0)$ & $\begin{array}{l}18.00(8.00 \\
98.00)\end{array}$ \\
\hline
\end{tabular}

T1: baseline; T2: 6 weeks to 3 months post-diagnosis; T3: 6 months to 1 year post-diagnosis; T4: 15 months to 2 years post-diagnosis. MET, metabolic equivalent task. 


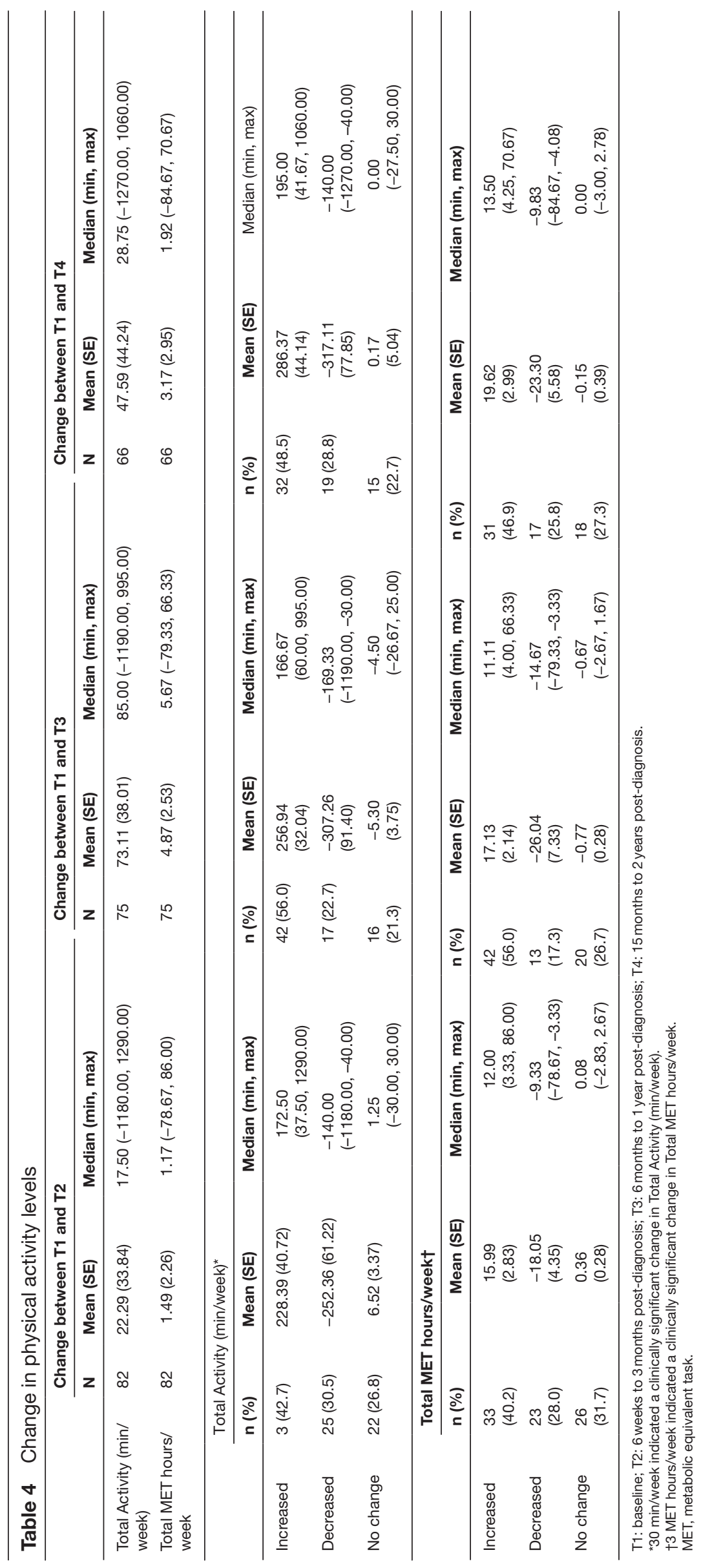

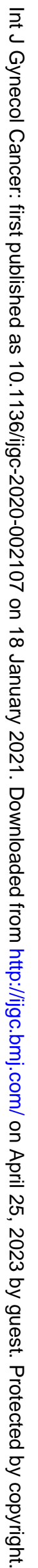




\section{Original research}

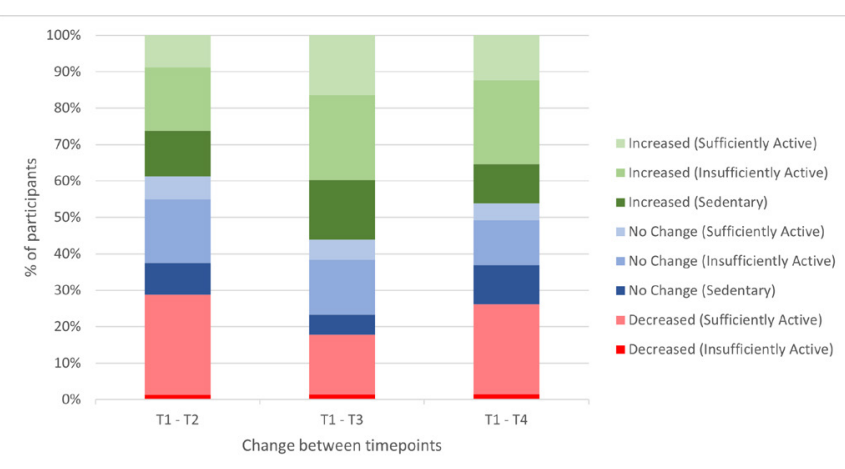

Figure 1 Clinically significant change in physical activity levels according to baseline physical activity category, where 3 MET hours/week indicates a clinically significant change. Sedentary: 0 MET hours/week; Insufficiently Active: $<7.5$ MET hours/week; Sufficiently Active: $\geq 7.5$ MET hours/week. T1: baseline; T2: 6 weeks to 3 months post-diagnosis; T3: 6 months to 1 year post-diagnosis; T4: 15 months to 2 years post-diagnosis. MET, metabolic equivalent task.

experienced clinically relevant changes in physical activity levels between T1 and T2-T4, according to T1 physical activity categories (ie, Sedentary, Insufficiently Active, or Sufficiently Active). Among women who were 'Sufficiently Active' at T1, approximately $60 \%$ had experienced clinically relevant declines in physical activity by T4. Additionally, among those who experienced a clinically relevant decrease in physical activity between T1 and T2-T4, over $90 \%$ were women who were 'Sufficiently Active' at $\mathrm{T1}$.

Table 5 reports participant characteristics associated with physical activity levels. Following adjustment for age and BMI (with higher BMI associated with lower levels of physical activity, $p=0.05$ ), diagnosis with stage IV disease $(p>0.05)$, lower income $(p>0.05)$, receipt of chemotherapy (adjuvant \pm neoadjuvant; $p<0.05$ ), smoking $(p<0.05)$, and currently working $(p<0.05)$ were associated with lower levels of physical activity. These same characteristics were associated with increased odds of being insufficiently active or sedentary. Lymph node dissection ( $p>0.05)$ was associated with higher physical activity levels and reduced odds of being insufficiently active or sedentary.

\section{DISCUSSION}

\section{Summary of main results}

The results from this prospective longitudinal cohort study suggest that, while approximately one in two women met physical activity guidelines post-ovarian cancer diagnosis, average physical activity levels for the cohort were below recommended levels. ${ }^{4-6}$ Additionally, by 2 years post-diagnosis, over half of the women reported either 'No Change' or a 'Decrease' in physical activity, with over $90 \%$ of those who reported a 'Decrease' having been 'Sufficiently Active' at baseline. Compared with those reporting higher levels of physical activity and meeting physical activity guidelines, women diagnosed with stage IV disease, those who were currently working, those earning a lower income, those receiving chemotherapy, and those who were currently smoking were more likely to report lower levels of physical activity and not meet physical activity guidelines.

\section{Results in the context of published literature}

In the current study, $53-57 \%$ of women reported being 'Sufficiently Active' (ie, meeting physical activity guidelines) post-diagnosis. This proportion is higher than previously observed, with several studies reporting that only $9-21 \%$ of women with ovarian cancer met physical activity guidelines between approximately 2-3 years post-diagnosis. ${ }^{922}{ }^{23}$ It is also higher than those previously observed in mixed (21-41\%; up to 21 years post-diagnosis), ${ }^{24} 25$ breast (21-40\%; 2-10 years post-diagnosis), ${ }^{26}$ and gynecological cancer cohorts $\left(9-53 \%\right.$; 6-24 months post-diagnosis). ${ }^{13}$ Differences in timing of assessment and study design may be contributing to these differences. Specifically, the study was longitudinal with prospective repeated measures of physical activity, whereas the broader evidence regarding physical activity post-cancer (particularly post-ovarian cancer) is derived from cross-sectional studies and/or retrospective data collection processes. ${ }^{14}$ Further, few studies involving gynecological cancer cohorts have assessed physical activity in the first year post-diagnosis. ${ }^{14}$

The findings reported here suggest that physical activity levels increased from baseline through to 2 years post-diagnosis assessment. However, our baseline physical activity assessment coincided with the point of diagnosis, a time typically associated with unresolved or heightened disease-related symptoms, pre-operative appointments and psychological stress, all of which would likely contribute to lower levels of baseline physical activity than would be observed in the year or decade prior to diagnosis. Consequently, it would be anticipated that resolution or stability of symptoms through treatment could contribute to the mean increase in physical activity levels observed. Also of note, however, less than half of the participants contributed to the mean increase observed over time; specifically, $27 \%$ and $26 \%$ of women reported either 'No Change' or a 'Decrease' in physical activity between baseline (at diagnosis) and 2 years post-diagnosis, respectively. Additionally, of those reporting a 'Decrease' in physical activity, over $90 \%$ had been 'Sufficiently Active' at baseline. Furthermore, overall average physical activity levels remained below the recommended $150 \mathrm{~min} /$ week throughout the entire 2-year follow-up. ${ }^{4-6}$ These findings are consistent with physical activity levels observed in other more commonly studied cancer cohorts, ${ }^{11}{ }^{126}$ and support the inclusion of women meeting physical activity guidelines in exercise intervention or physical activity programs, with the goal of maintaining physical activity post-diagnosis. Considering the benefits of physical activity and exercise on cancer-specific health outcomes, ${ }^{12}$ it is important to understand how the challenges experienced by women with ovarian cancer may impact physical activity levels and whether the current guidelines are appropriate for this specific cancer type. Combining this understanding with the literature examining physical activity preferences (eg, home-based, walking), barriers and identifying those at risk of physical inactivity would further improve our ability to provide appropriate physical activity advice. $^{2728}$

The findings from this study also suggest that several characteristics are associated with reporting lower levels of physical activity and increased odds of being insufficiently active or sedentary. These include being diagnosed with stage IV disease, receipt of chemotherapy, lower income, smoking, or currently working. Given the morbidity associated with treatment for more advanced disease (including more extensive surgery), it is possible that side effects 
Table 5 Characteristics associated with physical activity levels

\begin{tabular}{|c|c|c|c|c|c|c|c|c|c|}
\hline \multirow[b]{3}{*}{ Characteristics } & \multicolumn{5}{|c|}{$\begin{array}{l}\text { Continuous physical activity* } \\
\text { (Total MET hours/week) }\end{array}$} & \multicolumn{4}{|c|}{$\begin{array}{l}\text { Categorical physical activity* } \\
\text { (odds of being insufficiently active) }\end{array}$} \\
\hline & \multirow[b]{2}{*}{$\beta$} & \multirow[b]{2}{*}{ SE } & \multicolumn{2}{|c|}{$95 \%$ Wald $\mathrm{Cl}$} & \multirow[b]{2}{*}{$P$ value } & \multirow[b]{2}{*}{$\operatorname{Exp}(\beta)$} & \multicolumn{2}{|c|}{$\begin{array}{l}\text { 95\% Wald } \mathrm{Cl} \text { for Exp } \\
(\beta)\end{array}$} & \multirow[b]{2}{*}{$P$ value } \\
\hline & & & Lower & Upper & & & Lower & Upper & \\
\hline Age (years) & -0.08 & 0.10 & -0.29 & 0.12 & 0.42 & 1.02 & 0.99 & 1.04 & 0.30 \\
\hline Body mass index $\left(\mathrm{kg} / \mathrm{m}^{2}\right)$ & -0.35 & 0.17 & -0.69 & -0.01 & 0.05 & 1.01 & 0.96 & 1.06 & 0.73 \\
\hline
\end{tabular}

\begin{tabular}{|c|c|c|c|c|c|c|c|c|c|c|}
\hline & \multirow[b]{2}{*}{$\%$} & \multirow[b]{2}{*}{ EM Mean } & \multirow{2}{*}{$\begin{array}{l}\text { SE (EM } \\
\text { Mean) }\end{array}$} & \multicolumn{2}{|c|}{ 95\% Wald Cl } & \multirow[b]{2}{*}{$P$ value } & \multirow[b]{2}{*}{$\operatorname{Exp}(\beta)$} & \multicolumn{2}{|c|}{ 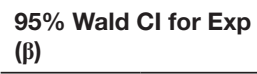 } & \multirow[b]{2}{*}{$P$ value } \\
\hline & & & & Lower & Upper & & & Lower & Upper & \\
\hline Stage Intercept & $\mathrm{n}=105$ & & & & & 0.00 & & & & 0.37 \\
\hline Stage & & & & & & 0.44 & & & & 0.48 \\
\hline Stage I & $27.70 \%$ & 14.80 & 2.38 & 10.15 & 19.46 & Ref & Ref & Ref & Ref & Ref \\
\hline Stage II & $12.40 \%$ & 12.21 & 3.02 & 6.29 & 18.12 & 0.49 & 1.25 & 0.45 & 3.46 & 0.67 \\
\hline Stage III & $47.30 \%$ & 15.98 & 2.12 & 11.83 & 20.12 & 0.71 & 0.99 & 0.50 & 1.95 & 0.97 \\
\hline Stage IV & $12.70 \%$ & 11.21 & 2.34 & 6.62 & 15.79 & 0.28 & 1.91 & 0.74 & 4.93 & 0.18 \\
\hline Income Intercept & $n=89$ & & & & & 0.01 & & & & 0.50 \\
\hline Income & & & & & & 0.39 & & & & 0.36 \\
\hline$>\$ 60000+$ & $21.70 \%$ & 12.02 & 3.01 & 6.13 & 17.91 & 0.39 & 1.51 & 0.62 & 3.66 & 0.36 \\
\hline$\leq \$ 60000$ & $78.30 \%$ & 15.12 & 1.68 & 11.83 & 18.41 & Ref & Ref & Ref & Ref & Ref \\
\hline Lymph node dissection Intercept & $\mathrm{n}=108$ & & & & & 0.01 & & & & 0.57 \\
\hline Lymph node dissection & & & & & & 0.05 & & & & 0.05 \\
\hline Yes & $39.70 \%$ & 17.46 & 1.92 & 13.69 & 21.24 & 0.05 & 0.56 & 0.31 & 0.99 & 0.05 \\
\hline No & $60.30 \%$ & 12.25 & 1.68 & 8.95 & 15.54 & Ref & Ref & Ref & Ref & Ref \\
\hline Chemotherapy Intercept & $\mathrm{n}=103$ & & & & & 0.00 & & & & 0.19 \\
\hline Chemotherapy & & & & & & 0.43 & & & & 0.32 \\
\hline Adjuvant \pm neoadjuvant & $81.90 \%$ & 14.18 & 1.45 & 11.33 & 17.03 & 0.43 & 1.48 & 0.69 & 3.20 & 0.32 \\
\hline No chemotherapy & $18.10 \%$ & 16.78 & 2.94 & 11.02 & 22.54 & Ref & Ref & Ref & Ref & Ref \\
\hline Smoking Intercept & $\mathrm{n}=104$ & & & & & 0.00 & & & & 0.46 \\
\hline Smoking & & & & & & 0.01 & & & & 0.06 \\
\hline Current & $10.40 \%$ & 9.26 & 1.70 & 5.92 & 12.60 & 0.01 & 2.07 & 0.98 & 4.33 & 0.06 \\
\hline Never/past & $89.60 \%$ & 15.07 & 1.43 & 12.27 & 17.87 & Ref & Ref & Ref & Ref & Ref \\
\hline Employment Intercept & $\mathrm{n}=102$ & & & & & 0.00 & & & & 0.21 \\
\hline Employment & & & & & & 0.22 & & & & 0.36 \\
\hline Other & $13.10 \%$ & 12.92 & 2.51 & 8.00 & 17.84 & 0.48 & 0.90 & 0.38 & 2.13 & 0.81 \\
\hline Retired/home duties & $62.80 \%$ & 16.42 & 1.87 & 12.75 & 20.09 & 0.09 & 0.60 & 0.29 & 1.27 & 0.18 \\
\hline Full-time/part-time/casual & $24.10 \%$ & 10.41 & 2.69 & 5.15 & 15.68 & Ref & Ref & Ref & Ref & Ref \\
\hline
\end{tabular}

*All available data at all time points contributed to the analysis.

EM mean, estimated marginal mean; MET, metabolic equivalent task.

associated with diagnosis of stage IV disease and receipt of chemotherapy would present barriers to participating in higher levels of physical activity and meeting recommended physical activity levels. ${ }^{4-6} 2930$ Lower socioeconomic status, time constraints, and participating in poor lifestyle behaviors have previously been associated with lower levels of physical activity in other cancer cohorts and chronic diseases. ${ }^{31-33}$ In the current analysis, lower income and currently working or smoking may represent potential surrogate measures for these characteristics. More difficult to understand was our observation that having lymph node dissection was associated with higher levels of physical activity and being less likely to be insufficiently active or sedentary. Women who received more extensive lymph node dissection (and consequently had a higher incidence of lower limb lymphedema) may have been referred to a lymphedema specialist, potentially being encouraged to participate in more activity as part of lymphedema treatment. ${ }^{1534}$ It is relevant to highlight that, while these results provide preliminary evidence supporting specific associations between patient, treatment and behavioral characteristics and physical activity levels, they represent partially-adjusted findings. A larger sample size would provide greater statistical power for a more in-depth analysis, with subsequent findings able to identify those most in need of physical activity support post-diagnosis.

\section{Implications for practice and future research}

The current evidence in support of physical activity (including exercise) post-cancer is largely based on studies that have involved more common cancer types with high survival rates, ${ }^{1235}$ with only 


\section{Original research}

preliminary (although supportive) evidence available in the ovarian cancer population. ${ }^{14}$ However, women with ovarian cancer typically have a poorer prognosis, more advanced disease at diagnosis, and require more extensive surgery and adjuvant treatment than the more common cancer types. ${ }^{36}{ }^{37}$ This study provides the first longitudinal evidence with prospective assessment of physical activity levels during a previously understudied time period (first year postdiagnosis of ovarian cancer). The subsequent findings suggest that within the first 2 years post-diagnosis of ovarian cancer, on average, women engage in physical activity levels below those recommended to cancer populations. Furthermore, $>50 \%$ either report no change in their physical activity or declines in their physical activity between diagnosis and 2 years post-diagnosis. Future research is now required to determine whether recommended levels are safe, feasible, and beneficial for this specific subgroup of the cancer population. A more in-depth understanding would also allow for the development of targeted physical activity and exercise recommendations that can accommodate the specific needs of the ovarian cancer population.

\section{Strengths and weaknesses of the study}

Strengths of this work include the prospective longitudinal design and 2-year follow-up, which clearly includes the previously understudied time frame of $<12$ months post-diagnosis and minimizes bias associated with retrospective and distant recall. ${ }^{38}{ }^{39}$ Additionally, the sample is representative of the wide cancer population, specifically with regard to age at diagnosis, stage and histological type of disease, and treatment patterns. ${ }^{3640}$ In contrast, study limitations include self-reported physical activity assessment (although using a validated instrument), which is subject to misinterpretation of exercise intensity and overestimation of physical activity levels, ${ }^{174142}$ and relatively small sample size preventing fullyadjusted regression analyses.

\section{CONCLUSIONS}

The findings from this work suggest that there is scope for women with ovarian cancer to benefit from participation in regular physical activity along with planned structured exercise. ${ }^{14}$ Additionally, the results from the current study demonstrate that average physical activity is below recommended guidelines, suggesting that there is capacity to positively influence physical activity levels in this cohort and potentially improve health, quality of life, and survival outcomes.

\author{
Author affiliations \\ ${ }^{1}$ School of Allied Health Sciences, Griffith University, Brisbane, Queensland, \\ Australia \\ ${ }^{2}$ Institute of Health and Biomedical Innovation, Queensland University of \\ Technology, Brisbane, Queensland, Australia \\ ${ }^{3}$ School of Exercise and Nutrition Sciences, Queensland University of Technology, \\ Brisbane, Queensland, Australia \\ ${ }^{4}$ Centre for Health Services Research, The University of Queensland, Brisbane, \\ Queensland, Australia \\ ${ }^{5}$ Queensland Centre for Gynaecological Cancer Research, The University of \\ Queensland, Brisbane, Queensland, Australia \\ ${ }^{6}$ Menzies Health Institute Queensland, Griffith University, Brisbane, Queensland, \\ Australia
}

Acknowledgements We would like to acknowledge the invaluable contribution of the women in the study; we are able to advance our understanding of ovarian cancer survivorship through their participation.
Contributors TLJ: conceptualization, methodology, software, validation, formal analysis, investigation, resources, data curation, writing original draft, visualization, project administration. CS: conceptualization, methodology, writing-review and editing, visualisation. DV: conceptualization, methodology, software, writingreview and editing. MJ: conceptualization, writing-reviewing and editing, funding acquisition. A0: conceptualization, writing-reviewing and editing, funding acquisition. SH: conceptualization, methodology, writing-reviewing and editing, visualization, supervision, funding acquisition.

Funding This work was supported by Cancer Australia (grant number 519711); Cancer Council Queensland (grant number 1026659); Wesley Research Foundation and the Gallipoli Foundation. We acknowledge Cancer Council Queensland for supporting SH through a Senior Research Fellowship and the National Health and Medical Research Council for supporting MJ through a Career Development Award (grant number 1045247).

Competing interests None declared.

\section{Patient consent for publication Not required.}

Ethics approval All procedures performed in studies involving human participants were in accordance with the ethical standards of the institutional and/or national research committee and with the 1964 Helsinki declaration and its later amendments or comparable ethical standards.

Provenance and peer review Not commissioned; externally peer reviewed.

Data availability statement Data are available upon reasonable request.

ORCID iD

Tamara Jones http://orcid.org/0000-0002-4854-0968

\section{REFERENCES}

1 Campbell KL, Winters-Stone KM, Wiskemann J, et al. Exercise guidelines for cancer survivors: consensus statement from international multidisciplinary roundtable. Med Sci Sports Exerc 2019;51:2375-90.

2 Hayes SC, Newton RU, Spence RR, et al. The Exercise and Sports Science Australia position statement: exercise medicine in cancer management. J Sci Med Sport 2019;22:1175-99.

3 Caspersen CJ, Powell KE, Christenson GM. Physical activity, exercise, and physical fitness: definitions and distinctions for healthrelated research. Public Health Rep 1985;100:126-31.

4 World Health Organization. Global recommendations on physical activity for health World Health Organization; 2010.

5 US Department of Health and Human Services. Physical activity guidelines for Americans. 2nd edn. Wasington, DC: US Dept of Health and Human Services, 2018.

6 Rock CL, Doyle C, Demark-Wahnefried W, et al. Nutrition and physical activity guidelines for cancer survivors. CA Cancer J Clin 2012;62:242-74.

7 Cormie P, Atkinson M, Bucci L, et al. Clinical Oncology Society of Australia position statement on exercise in cancer care. Med $\mathrm{J}$ Aust 2018;209:184-7.

8 Friedenreich CM, Stone CR, Cheung WY, et al. Physical activity and mortality in cancer survivors: a systematic review and meta-analysis. JNCl Cancer Spectr 2020;4.

9 Abbott SE, Camacho F, Peres LC, et al. Recreational physical activity and survival in African-American women with ovarian cancer. Cancer Causes Control 2018;29:77-86.

10 Irwin ML, Smith AW, McTiernan A, et al. Influence of pre- and postdiagnosis physical activity on mortality in breast cancer survivors: the health, eating, activity, and lifestyle study. J Clin Oncol 2008;26:3958-64.

11 De Groef A, Geraerts I, Demeyer H, et al. Physical activity levels after treatment for breast cancer: two-year follow-up. Breast 2018;40:23-8.

12 Fassier P, Zelek L, Partula V, et al. Variations of physical activity and sedentary behavior between before and after cancer diagnosis: results from the prospective population-based NutriNet-Santé cohort. Medicine 2016;95:e4629.

13 Lin K-Y, Edbrooke L, Granger CL, et al. The impact of gynaecological cancer treatment on physical activity levels: a systematic review of observational studies. Braz J Phys Ther 2019;23:79-92.

14 Jones TL, Sandler CX, Spence RR, et al. Physical activity and exercise in women with ovarian cancer: a systematic review. Gynecol Oncol 2020;158:803-11.

15 Hayes SC, Janda M, Ward LC, et al. Lymphedema following gynecological cancer: results from a prospective, longitudinal cohort 
study on prevalence, incidence and risk factors. Gynecol Oncol 2017;146:623-9.

16 DiSipio T, Janda M, Hayes S, et al. The Lymphedema Evaluation in Gynecological cancer Study (LEGS): design of a prospective, longitudinal, cohort study. Cancer Research Frontiers 2015;1:104-18.

17 Australian Institute of Health and Welfare. The Active Australia Survey: a guide and manual for implementation, analysis and reporting. Canberra: AlHW, 2003.

18 Ainsworth BE, Haskell WL, Whitt MC, et al. Compendium of physical activities: an update of activity codes and MET intensities. Med Sci Sports Exerc 2000;32:S498-516.

19 Li T, Wei S, Shi Y, et al. The dose-response effect of physical activity on cancer mortality: findings from 71 prospective cohort studies. $\mathrm{Br}$ J Sports Med 2016:50:339-45.

20 Hair BY, Hayes S, Tse C-K, et al. Racial differences in physical activity among breast cancer survivors: implications for breast cancer care. Cancer 2014;120:2174-82.

21 Harrison S, Hayes SC, Newman B. Level of physical activity and characteristics associated with change following breast cancer diagnosis and treatment. Psychooncology 2009;18:387-94.

22 Mizrahi D, Naumann F, Broderick C, et al. Quantifying physical activity and the associated barriers for women with ovarian cancer Int J Gynecol Cancer 2015;25:577-83.

23 Smits A, Smits E, Lopes A, et al. Body mass index, physical activity and quality of life of ovarian cancer survivors: time to get moving? Gynecol Oncol 2015;139:148-54.

24 Coups EJ, Ostroff JS. A population-based estimate of the prevalence of behavioral risk factors among adult cancer survivors and noncancer controls. Prev Med 2005;40:702-11.

25 Eakin EG, Youlden DR, Baade PD, et al. Health behaviors of cancer survivors: data from an Australian population-based survey. Cancer Causes Control 2007;18:881-94.

26 Mason C, Alfano CM, Smith AW, et al. Long-term physical activity trends in breast cancer survivors. Cancer Epidemiol Biomarkers Prev 2013;22:1153-61.

27 Stevinson C, Capstick V, Schepansky A, et al. Physical activity preferences of ovarian cancer survivors. Psychooncology 2009;18:422-8.

28 Stevinson C, Tonkin K, Capstick V, et al. A population-based study of the determinants of physical activity in ovarian cancer survivors. $J$ Phys Act Health 2009;6:339-46.
29 Arriba LN, Fader AN, Frasure $\mathrm{HE}$, et al. A review of issues surrounding quality of life among women with ovarian cancer. Gynecol Oncol 2010;119:390-6.

30 Keim-Malpass J, Mihalko SL, Russell G, et al. Problems experienced by ovarian cancer survivors during treatment. J Obstet Gynecol Neonatal Nurs 2017;46:544-54.

31 Clifford BK, Mizrahi D, Sandler CX, et al. Barriers and facilitators of exercise experienced by cancer survivors: a mixed methods systematic review. Support Care Cancer 2018;26:685-700.

32 Naik H, Qiu X, Brown MC, et al. Socioeconomic status and lifestyle behaviours in cancer survivors: smoking and physical activity. Curr Oncol 2016;23:546-55.

33 Gjerset GM, Fosså SD, Courneya KS, et al. Exercise behavior in cancer survivors and associated factors. J Cancer Surviv 2011;5:35-43.

34 Borman P, diagnosis L. Lymphedema diagnosis, treatment, and follow-up from the view point of physical medicine and rehabilitation specialists. Turk J Phys Med Rehabil 2018;64:179-97.

35 Australian Institute of Health and Welfare. Cancer in Australia 2019. Canberra: AlHW, 2019

36 Australian Institute of Health and Welfare. Ovarian cancer in Australia: an overview, 2010. Canberra: AlHW, 2010.

37 Jelovac D, Armstrong DK. Recent progress in the diagnosis and treatment of ovarian cancer. CA Cancer J Clin 2011;61:183-203.

38 Falkner KL, Trevisan M, McCann SE. Reliability of recall of physical activity in the distant past. Am J Epidemiol 1999;150:195-205.

39 Blair SN, Dowda M, Pate RR, et al. Reliability of long-term recall of participation in physical activity by middle-aged men and women. Am J Epidemiol 1991;133:266-75.

40 Jessmon P, Boulanger T, Zhou W, et al. Epidemiology and treatment patterns of epithelial ovarian cancer. Expert Rev Anticancer Ther 2017; 17:427-37.

41 Bauman A, Merom D. Measurement and surveillance of physical activity in Australia - an introductory guide. Australasian Epidemiologist 2002;9:2-6.

42 Godino JG, Watkinson C, Corder K, et al. Awareness of physical activity in healthy middle-aged adults: a cross-sectional study of associations with sociodemographic, biological, behavioural, and psychological factors. BMC Public Health 2014;14:421. 